\title{
Precision Measurements of the Proton Elastic Form Factor Ratio
}

\author{
D. W. Higinbotham \\ Jefferson Lab, Newport News, VA 23606, USA
}

\begin{abstract}
New high precision polarization measurements of the proton elastic form factor ratio in the $\mathrm{Q}^{2}$ from 0.3 to $0.7[\mathrm{GeV} / \mathrm{c}]^{2}$ have been made. These elastic $\mathrm{H}(\mathrm{e}, \mathrm{e}$ 'p $)$ measurements were done in Jefferson Lab's Hall A using $80 \%$ longitudinally polarized electrons and recoil polarimetry. For $\mathrm{Q}^{2}$ greater than $1[\mathrm{GeV} / \mathrm{c}]^{2}$, previous polarization data indicated a strong deviation of the form factor ratio from unity which sparked renewed theoretical and experimental interest in how two-photon diagrams have been taken into account. The new high precision data indicate that the deviation from unity, while small, persists even at $\mathrm{Q}^{2}$ less than $1[\mathrm{GeV} / \mathrm{c}]^{2}$.
\end{abstract}

Keywords: Proton Form Factor

PACS: 14.20.Dh,13.85.Dz,13.88.+e

\section{INTRODUCTION}

Understanding the four-momentum transfer squared, $\mathrm{Q}^{2}$, dependence of the proton's electro-magnetic form factors is fundamental to understanding the proton and as well as a necessary input parameter in many calculations. Cross section measurements generally show that the ratio of the proton's electric to magnetic form factor is basically unity [1], while at $\mathrm{Q}^{2}>1[\mathrm{GeV} / \mathrm{c}]^{2}$ recoil polarization measurements $[2,3,4,5]$ as well a beam-target polarization measurement [6] have observed a deviation from unity. At this time, the most likely cause for the difference between the cross section results and the polarization results is the two-photon part of the radiative corrections [7].

\section{RADIATIVE CORRECTIONS}

It is important to understand that two-photon diagrams have been included in the standard cross section radiative corrections such as Mo and Tsai [8], but they have been included with varying degrees of approximation. For example, the Feynman diagrams for Mo and Tsai's approach are shown in Fig. 1 where the proton's structure is neglected and the two-photons were only allowed to have either all the four-momentum transfer or none.

Recent calculations of radiative corrections not only integrate over all possible photon energies and the proton's structure, but even allow the proton within the box-diagrams to be off-shell as shown in Fig. 2. These effects make the calculations particularly challenging as it is the proton's structure that one is trying to determine $[9,10,11]$ In fact, the unexpected discrepancy in the cross section and asymmetry measurements should, in the long run, dramatically improve our understanding of not only radiative 


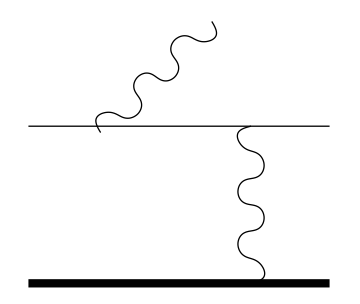

Bremsstrahlung
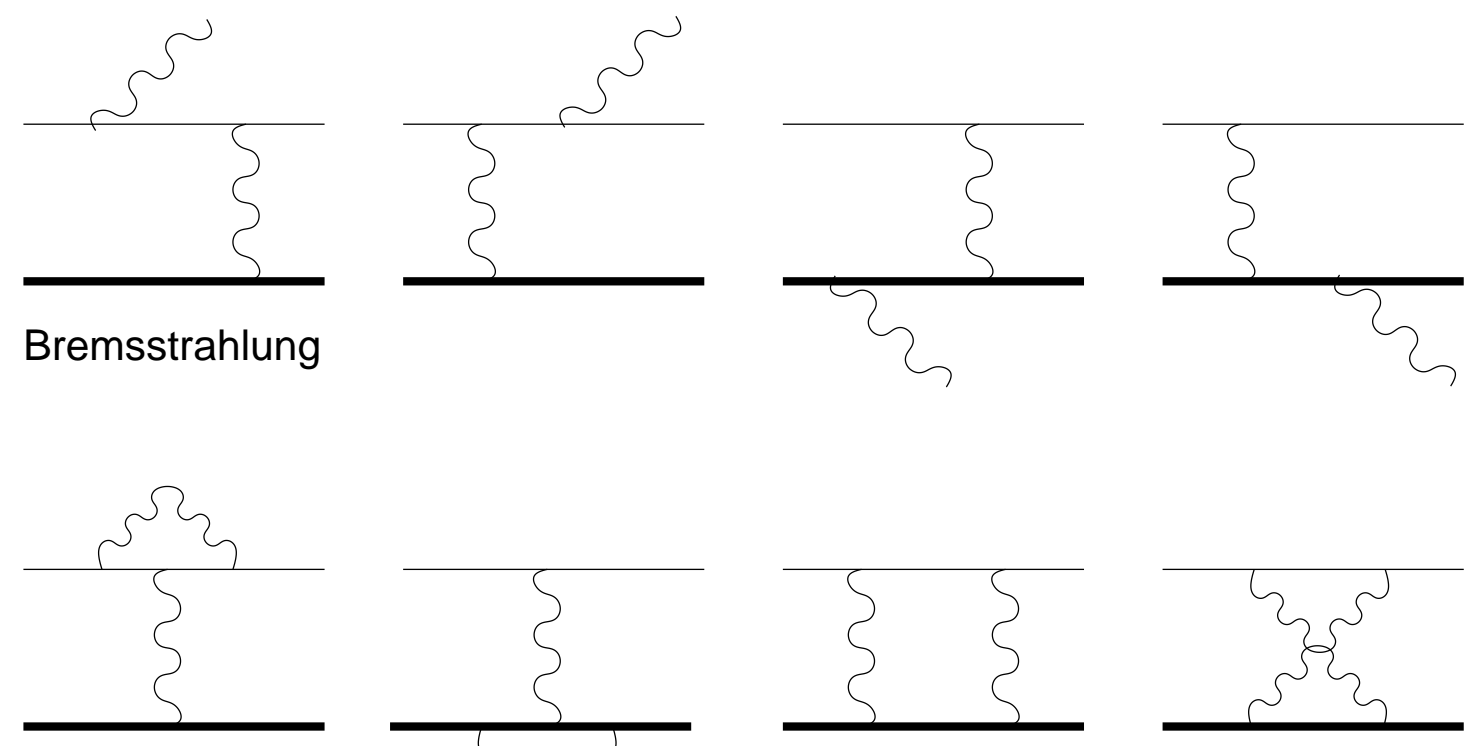

Vertex Corrections

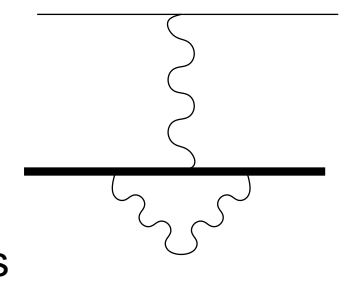

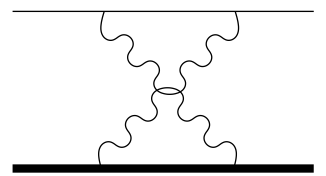

Box Diagrams

FIGURE 1. Shown are the standard Feynman diagrams included in the calculation of radiative corrections in a lepton-hadron scattering experiment. The thin line represents the lepton, the thick line the hadron and the wavy line(s) the virtual photon(s).

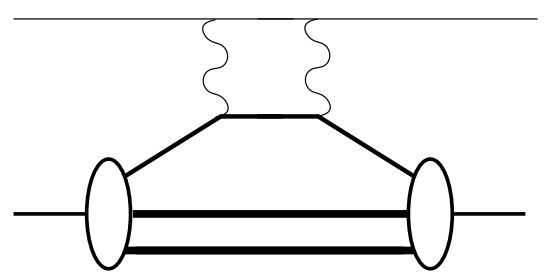

Handbag Diagram

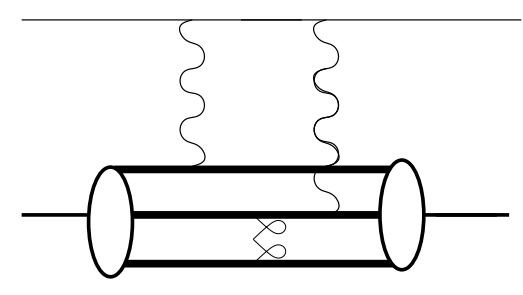

pQCD Diagram

FIGURE 2. Shown are the handbag and pQCD diagrams for handling the two-photon corrections in a more detailed picture. In the handbag diagram the virtual photons couple to a single parton, while in the pQCD diagram, the virtual photons couple to different partons with a gluon coupling the last two partons together.

corrections, but also the proton's structure.

\section{LOW $Q^{2}$ FORM FACTOR MEASUREMENTS}

The observed discrepancy between cross section and asymmetry measurements as well as the calculation of the two-photon corrections has focused on $Q^{2}>1[\mathrm{GeV} / \mathrm{c}]^{2}$. Previous low $\mathrm{Q}^{2}$ polarization measurements $[12,13]$ perhaps saw hints of an effect for $\mathrm{Q}^{2}<1[\mathrm{GeV} / \mathrm{c}]^{2}$, but certainly nothing definitive. Experiment E08-007 at Jefferson Lab [14] made a high precision survey of the form factor ratio in the $\mathrm{Q}^{2}$ range from 
0.3 to $0.7[\mathrm{GeV} / \mathrm{c}]^{2}$ using the recoil polarization technique. The experiment was done in Hall A [15] with a High Resolution Spectrometer (HRS) detecting the elastically scattered proton and the BigBite magnet $[16,17]$ along with a lead glass calorimeter used for tagging the elastic electrons. In addition, a $6 \mathrm{~cm}$ long liquid hydrogen target was used along with $80 \%$ longitudinally polarized electrons from the Continuous Electron Beam Accelerator Facility (CEBAF) [18].

As knowledge of the spin precession within the high resolution spectrometer is critical for the extraction of the form factors, three different HRS momentum settings were used for each of the kinematics shown in Table 1. This was possible since the nominal momentum bite of the HRS is $\pm 4.5 \%$, so by choosing momenta of $0 \%$ and $\pm 2 \%$ around the nominal elastic kinematic settings, we changed the spin precession while still staying well within the nominal acceptance of the device. Each of these settings was also measured to $1-2 \%$ statistics so that systematic effects could be studied and not confused with statical fluctuations. It is worth noting that spin precession of spin-1/2 particles though a dipole magnetic field is extremely well understood; it is possible to calculate the thousands of degrees of spin precession the polarized electrons at CEBAF undergo as they travel five times around the accelerator [19]. In order to also take into account the effects of the super-conducting quadrupoles in the HRS, COSY, a spin transport modelling program, is used. For events with rays that pass near the central ray of the spectrometer, the COSY model and the simple dipole model agree. Without using COSY, as one goes away from the central ray in angle, a strong slope can be seen in the form factor ratio. This slope goes away once the COSY spin matrix is applied.

The preliminary results of this experiment indicate that the deviation of the ratio of the form factor smoothly continues into the low $\mathrm{Q}^{2}$ and that there is no sharp transition to unity around $\mathrm{Q}^{2}$ equal to one. It does appear that a rapid change in the ratio must occur for the $\mathrm{Q}^{2}$ less then $0.3[\mathrm{GeV} / \mathrm{c}]^{2}$ either with a rise to zero such as in relativistic pQCD models [20] or with a rise above one near $\mathrm{Q}^{2}$ of $0.1[\mathrm{GeV} / \mathrm{c}]^{2}$ and then a return to unity at a $\mathrm{Q}^{2}$ of zero.

TABLE 1. Shown are the central angles and momentum for the high resolution spectrometer used for proton detection and the kinematics for the BigBite spectrometer used for electron detection. For each $Q^{2}$, three different spectrometer momentum settings were used; all within the nominal momentum acceptance of the spectrometer.

\begin{tabular}{ccccc}
\hline$Q^{2}[\mathbf{G e V} / \mathbf{c}]^{2}$ & $\theta_{p}[\mathbf{d e g}]$ & $P_{p}[\mathbf{G e V} / \mathbf{c}]$ & $\theta_{e}[\mathbf{d e g}]$ & $P_{e}[\mathbf{G e V} / \mathbf{c}]$ \\
\hline 0.30 & 60.0 & 0.565 & 30.0 & 1.03 \\
0.35 & 57.5 & 0.616 & 30.0 & 1.01 \\
0.41 & 55.0 & 0.668 & 35.0 & 0.978 \\
0.45 & 53.0 & 0.710 & 35.0 & 0.954 \\
0.50 & 51.0 & 0.752 & 40.0 & 0.928 \\
0.55 & 49.0 & 0.794 & 40.0 & 0.901 \\
0.60 & 47.0 & 0.836 & 45.0 & 0.874 \\
0.70 & 43.5 & 0.913 & 50.0 & 0.823 \\
\hline
\end{tabular}




\section{NUCLEONS IN DEUTERIUM}

The simplest system in which to study a bound nucleon is the deuteron. Polarized beam and vector polarized target experiments at NIKHEF showed that at low $\mathrm{Q}^{2}$ the $\mathrm{D}\left(\mathrm{e}, \mathrm{e}^{\prime} \mathrm{p}\right) \mathrm{n}$ reaction asymmetry was the same as the $\mathrm{H}\left(\mathrm{e}, \mathrm{e}^{\prime} \mathrm{p}\right)$ elastic asymmetry and only at missing momentum greater than approximately $100[\mathrm{MeV} / \mathrm{c}]$ was an appreciable deviation observed [21]. The MIT-Bates recoil polarization experiment also didn't see any large difference between hydrogen elastic and low missing momentum deuteron quasi-elastic scattering $[22,23]$. This seems to contradict the recoil polarization results of B. Hu et al. [24] where a deviation was reported. Since the data of B. Hu was taken with the same equipment as the new high precision hydrogen data, it is straightforward to include the new results and look at the ratios again as shown in Table 2 . By doing this, the $\chi^{2} / n$ of the hypothesis of a ratio of one goes from 1.7 to 0.7 . Thus, within experimental uncertainties, the low missing momentum data are consistent with no effect. It is now clear that what drove the original deviation was a high free proton form factor ratio. The current result again validates the common technique of extracting neutron properties from reactions on neutrons in a deuteron target, with small theoretical corrections.

TABLE 2. Shown is the results from B. Hu [24] along with the new proton form factor data from E08-007. By including the new data, the $\chi^{2} / n$ of a flat line fit of the ratio goes from 1.7 to 0.7 ; thus indicating the low $P_{m}$ (e,e'p) reaction from deuterium is consistent with the free proton case. Do note that for the high $Q^{2}$ point of 1.61 , there are large systematics errors in determining the individual ratios that cancel in the ratio.

\begin{tabular}{cccccc}
\hline $\begin{array}{c}Q^{2} \\
{[\mathbf{G e V} / \mathbf{c}]^{2}}\end{array}$ & $\begin{array}{c}\text { Deuteron } \\
\mu G_{E} / G_{M}\end{array}$ & $\begin{array}{c}\text { Proton } \\
\mu G_{E} / G_{M}\end{array}$ & $\begin{array}{c}\text { Old Ratios } \\
P_{D} / P_{\text {free }}\end{array}$ & $\begin{array}{c}\text { New Proton } \\
\mu G_{E} / G_{M}\end{array}$ & $\begin{array}{c}\text { New Ratio } \\
P_{D} / P_{\text {free }}\end{array}$ \\
\hline 0.43 & $0.92 \pm 0.03$ & $0.99 \pm 0.03$ & $0.92 \pm 0.04$ & $0.93 \pm 0.01$ & $0.99 \pm 0.03$ \\
1.00 & $0.88 \pm 0.01$ & $0.88 \pm 0.02$ & $1.00 \pm 0.03$ & & \\
1.61 & $0.93 \pm 0.04$ & $0.87 \pm 0.04$ & $1.08 \pm 0.07$ & & \\
\hline
\end{tabular}

\section{FUTURE EXPERIMENTS}

It would be very interesting to push high precision proton form factor measurements to lower $\mathrm{Q}^{2}$, but the technique of recoil polarization requires protons with reasonable momenta, so it is not feasible to go to lower $\mathrm{Q}^{2}$ with the setup described herein. With an electron-proton collider, it would be possible to get to lower $\mathrm{Q}^{2}$ due to the boost of the proton's momentum, or one can do the measurement with a polarized beam and a fixed target where only the scattered electron needs to be detected. Such an experiment is planned for 2012 at Jefferson Lab as the second half of experiment E08-007 [14] and will cover the $\mathrm{Q}^{2}$ range from 0.015 to $0.4[\mathrm{GeV} / \mathrm{c}]^{2}$.

If two-photon diagrams are truly the solution to the discrepancy between the cross section and the asymmetry experiments, this should be clearly seen in the upcoming high precision electron-proton and positron-proton cross section ratio experiments such as the Jefferson Lab's Hall B experiment [25] presented herein by B. Raue (FIU), the VEPP-III experiment [26] and the Olympus experiment at DESY [27]. 


\section{ACKNOWLEDGMENTS}

I thank Carl Carlson (W\&M) for teaching me about the history of two-photon corrections and Ph.D. student Xiaohui Zhan (MIT) for her outstanding work analyzing the new low $\mathrm{Q}^{2}$ data. This work was supported by the U.S. Department of Energy, and Jefferson Science Associates which operates the Thomas Jefferson National Accelerator Facility under DOE contract DE-AC05-06OR23177.

\section{REFERENCES}

1. I. A. Qattan, et al., Phys. Rev. Lett. 94, 142301 (2005).

2. M. K. Jones, et al., Phys. Rev. Lett. 84, 1398-1402 (2000).

3. O. Gayou, et al., Phys. Rev. Lett. 88, 092301 (2002).

4. O. Gayou, et al., Phys. Rev. C64, 038202 (2001).

5. V. Punjabi, et al., Phys. Rev. C71, 055202 (2005).

6. M. K. Jones, et al., Phys. Rev. C74, 035201 (2006).

7. P. A. M. Guichon, and M. Vanderhaeghen, Phys. Rev. Lett. 91, 142303 (2003).

8. L. W. Mo, and Y.-S. Tsai, Rev. Mod. Phys. 41, 205-235 (1969).

9. A. V. Afanasev, S. J. Brodsky, C. E. Carlson, Y.-C. Chen, and M. Vanderhaeghen, Phys. Rev. D72, 013008 (2005).

10. P. G. Blunden, W. Melnitchouk, and J. A. Tjon, Phys. Rev. C72, 034612 (2005).

11. N. Kivel, and M. Vanderhaeghen, Phys. Rev. Lett. 103, 092004 (2009).

12. C. B. Crawford, et al., Phys. Rev. Lett. 98, 052301 (2007).

13. G. Ron, et al., Phys. Rev. Lett. 99, 202002 (2007).

14. J. Arrington, D. Day, R. Gilman, D. Higinbotham, G. Ron, and A. Sarty, E08-007: The proton form factor ratio at low $\mathrm{Q}^{2}$ (2008), Jefferson Lab.

15. J. Alcorn, et al., Nucl. Instrum. Meth. A522, 294-346 (2004).

16. D. J. J. de Lange, et al., Nucl. Instrum. Meth. A406, 182-194 (1998).

17. D. J. J. de Lange, et al., Nucl. Instrum. Meth. A412, 254-264 (1998).

18. C. W. Leeman, D. R. Douglas, and G. A. Krafft, Annu. Rev. Nucl. Part. Sci. 51, 413 (2001).

19. D. W. Higinbotham, AIP Conf. Proc. 1149, 751-754 (2009), 0901 . 4484.

20. J. P. B. C. de Melo, T. Frederico, E. Pace, S. Pisano, and G. Salme, Physics Letters B 671, 153 (2009).

21. I. Passchier, et al., Phys. Rev. Lett. 82, 4988-4991 (1999).

22. B. D. Milbrath, et al., Phys. Rev. Lett. 80, 452-455 (1998).

23. B. D. Milbrath, et al., Phys. Rev. Lett. 82, 2221 (1999).

24. B. Hu, et al., Phys. Rev. C73, 064004 (2006).

25. W. Brooks, J. Arrington, K. Joo, B. Raue, A. Afanasev, and L. Weinstein, E04-116: A precise comparison of positron-proton and electron-proton elastic scattering in CLAS (2004), Jefferson Lab.

26. J. Arrington, et al. (2004), nucl-ex/ 0408020.

27. M. Kohl, AIP Conf. Proc. 1160, 19-23 (2009). 